\title{
Pd-catalyzed cyclodimerization of alkenyl and aryl dibromides: Construction of dibenzo[a,e]cyclooctatetraenes
}

\author{
Kunbing Ouyang a,b, Zhenfeng Xi \\ a Beijing National Laboratory for Molecular Sciences, Institute of Chemistry, Chinese Academy of Sciences, Beijing 100190, China \\ ${ }^{\mathrm{b}}$ Beijing National Laboratory for Molecular Sciences, Key Laboratory of Bioorganic Chemistry and Molecular Engineering of Ministry of Education, \\ College of Chemistry, Peking University, Beijing 100871, China
}

\section{A R T I C L E I N F O}

\section{Article history:}

Received 30 June 2014

Accepted 21 July 2014

Published 20 January 2015

\section{Keywords:}

Dibromide

Dibenzo[a,e]cyclooctatetraene

1,4-Dibromo-1,3-butadiene

Homo-coupling

Palladium catalyst
Cyclooctatetraene

\section{A B S T R A C T}

Cyclooctatetraenes, including dibenzocyclooctatetraenes, are structurally interesting compounds and are widely used in many areas. Therefore, the development of synthetic methods for such compounds has long been attractive. In this work, a Pd-catalyzed coupling reaction between alkenylbromides and arylbromides has been realized. Multi-substituted 1,4-dibromo-1,3-butadienes and $o$-bromo-2-(2-bromovinyl)benzenes underwent a Pd-catalyzed cyclodimerization reaction to afford the corresponding multi-substituted cyclooctatetraenes and dibenzo[a,e]cyclooctatetraenes, respectively, in high yields and with excellent regioselectivity.

(C) 2015, Dalian Institute of Chemical Physics, Chinese Academy of Sciences. Published by Elsevier B.V. All rights reserved.

\section{Introduction}

Multi-substituted cyclooctatetraenes (COTs) and their derivatives such as dibenzocyclooctatetraenes are a class of structurally interesting molecules that are useful organic intermediates [1-6] and sterically demanding ligands for metals [7-10]. Although several general and efficient methods are known for the preparation of multi-substituted COTs [11-17], many of these methods require a stoichiometric amount of lithium [15] or copper reagent [18-25]. Transition metal-catalyzed approaches for multi-substituted COTs mainly focus on the reaction of alkynes [26,27] tricyclo[4.2.0.02,5]octa3,7-dienes [28] and biphenylene derivatives [29-33].

Alkenyl dibromides have become important building blocks in organic synthesis [34-56]. They have been used to synthesize $\pi$-conjugated compounds via their corresponding lithium reagents [41-44], and pyrrole derivatives via a transition metal-catalyzed direct amination reaction [45-50]. We and others have developed efficient synthetic methods for dibromides o-bromo-2-(2-bromovinyl)benzenes (1) and 1,4-dibromo-1,3butadienes (2) (Scheme 1) [35,50-52]. Compounds such as these can be used in the synthesis of novel organometallic and organic compounds by taking advantage of their co-operative effect [22,53-56].

In principle, cyclic dimerization of functionalized dibromides $\mathbf{1}$ or $\mathbf{2}$ would afford cyclooctatetraenes and their derivatives. Such a strategy has been applied by Takahashi et al. [18], Itoh et al. [23,24], Xi et al. [25] and our group [19-22,57] when

\footnotetext{
*Corresponding author. Tel: +86-10-62759728; Fax: +86-10-62751708; E-mail: zfxi@pku.edu.cn

This work was supported by the National Basic Research Program of China (973 Program, 2012CB821600) and the National Natural Science Foundation of China (21132001). 


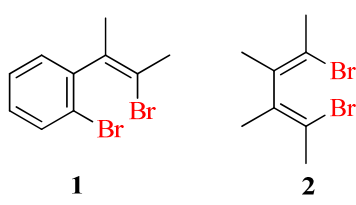

Previous work: stoichiometric reaction
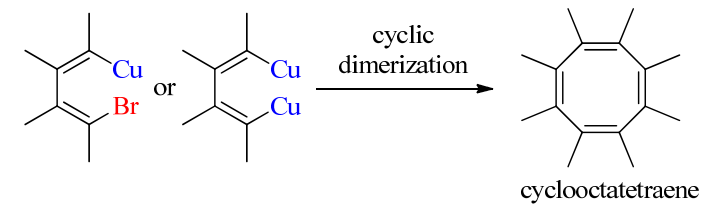

Previous work: catalytic reaction
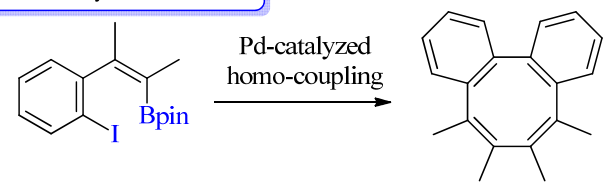

dibenzo[a,c]-cyclooctatetraene

This work: catalytic reaction

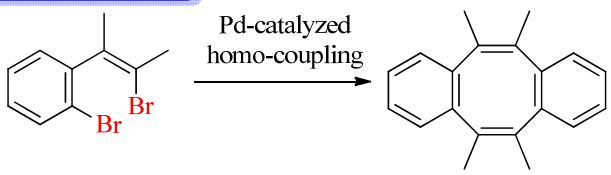

dibenzo[a,e]-cyclooctatetraene

Scheme 1. Synthesis of cyclooctatetraenes and their derivatives from alkenyl and aryl dibromides.

one or two of the halogen atoms are replaced by $\mathrm{Cu}$ or Bpin (Scheme 1). In this work, we would like to report the first catalytic and direct homo-coupling of dibromides $\mathbf{1}$ or $\mathbf{2}$ for the synthesis of dibenzo[a,e]cyclooctatetraenes and cyclooctatetraenes (Scheme 1).

\section{Experimental}

\subsection{General}

Unless otherwise noted, all starting materials were commercially available and were used without further purification. All reactions were carried out using standard Schlenk techniques or under a nitrogen atmosphere in a glovebox. The nitrogen in the glovebox was constantly circulated through a copper/molecular sieve catalyst unit. The oxygen and moisture concentrations in the glovebox atmosphere were monitored by an $\mathrm{O}_{2} / \mathrm{H}_{2} \mathrm{O}$ Combi-Analyzer to ensure both were always below $1 \mathrm{ppm}$. Solvents were purified by an Mbraun SPS-800 Solvent Purification System and dried over fresh Na chips in the glovebox. ${ }^{1} \mathrm{H}$ and ${ }^{13} \mathrm{C}$ NMR spectra were recorded on a Bruker ARX 400 spectrometer (FT, $400 \mathrm{MHz}$ for ${ }^{1} \mathrm{H}$; $100 \mathrm{MHz}$ for ${ }^{13} \mathrm{C}$ ) or a Bruker AVANCE III 500 spectrometer (FT, $500 \mathrm{MHz}$ for ${ }^{1} \mathrm{H}$; 125 $\mathrm{MHz}$ for ${ }^{13} \mathrm{C}$ ) at room temperature in a $\mathrm{CDCl}_{3}$ solution with tetramethylsilane $(0.00 \mathrm{ppm})$ as the internal standard, unless otherwise noted. High-resolution mass spectra (HRMS) were recorded on a Bruker Apex IV FTMS mass spectrometer using ESI and FT-ICR mass analyzer or a Bruker Daltonics Inc APEXII mass spectrometer using EI and FT-ICR mass analyzer. GC/MS analyses were recorded on Agilent 7890A/5975C using EI MSD.

\subsection{Preparation of dibenzocyclooctatetraenes and octaethylcyclooctatetraene}

The dibromo-substituted vinyl compounds $\mathbf{1}$ and $\mathbf{2}$ were prepared according to literature methods [50-52]. A dibromo-substituted substrate (0.6 mmol, 2 eq. $), \operatorname{Pd}\left(t-\mathrm{Bu}_{3} \mathrm{P}\right)_{2}(0.015$ mmol, 5 mol\%), $t$-Bus P (0.03 mmol, $10 \mathrm{~mol} \%$ ), and LiOEt (1.2 mmol, 4 eq.) were added to $5 \mathrm{~mL}$ toluene. The mixture was stirred at $120{ }^{\circ} \mathrm{C}$ for $24 \mathrm{~h}$. The reaction mixture was quenched with water and extracted with ethyl acetate. The organic extract was washed with brine and dried over $\mathrm{Na}_{2} \mathrm{SO}_{4}$. The solvent was then evaporated in vacuo and the residue was purified using a silica gel column, eluting with petroleum ether and ethyl acetate, to afford the multi-substituted cyclooctatetraenes $\mathbf{3}$ and 4.

\subsection{Analytical data for the dibromides $\mathbf{1}$}

(Z)-1-Bromo-2-(3-bromobut-2-en-2-yl)benzene (1c). White solid, isolated yield 95\% (547 mg); m.p: $33.8-34.7{ }^{\circ} \mathrm{C}$; ${ }^{1} \mathrm{H} \mathrm{NMR}$ (400 MHz, $\left.\mathrm{CDCl}_{3}\right) \delta: 1.93(\mathrm{~s}, 3 \mathrm{H}), 2.37(\mathrm{~s}, 3 \mathrm{H}), 7.02-7.06(\mathrm{~m}$, $2 \mathrm{H}), 7.21(\mathrm{t}, J=7.5 \mathrm{~Hz}, 1 \mathrm{H}), 7.51(\mathrm{~d}, J=8.1 \mathrm{~Hz}, 1 \mathrm{H}) ;{ }^{13} \mathrm{C} \mathrm{NMR}$ $\left(100 \mathrm{MHz}_{\mathrm{CDCl}}\right) \delta: 20.16\left(1 \mathrm{CH}_{3}\right), 24.77\left(1 \mathrm{CH}_{3}\right), 118.68$ (1 quat. C), 121.61 (1 quat. C), $127.27(1 \mathrm{CH}), 128.26(1 \mathrm{CH})$, 129.30 (1 CH), 132.36 (1 CH), 135.05 (1 quat. C), 145.04 (1 quat. C); HRMS (ESI, $m / z$ ) calcd. for $\mathrm{C}_{10} \mathrm{H}_{11} \mathrm{Br}_{2} \quad[\mathrm{M}+\mathrm{H}]^{+}$: 288.9222; Found 288.9219.

(Z)-1-Bromo-2-(1-bromo-1-phenylbut-1-en-2-yl)benzene (1d). White solid, isolated yield 85\% (619 mg); m.p: 34.9-38.31 ${ }^{\circ} \mathrm{C}$; ${ }_{1}^{1} \mathrm{H} \mathrm{NMR} \mathrm{(400} \mathrm{MHz,} \mathrm{CDCl}_{3}$ ) $\delta: 0.91(\mathrm{t}, J=7.5 \mathrm{~Hz}, 3 \mathrm{H}$ ), 2.29-2.44 (m, 2H), 7.12-7.34 (m, 5H), 7.36-7.42 (m, 2H), 7.45-7.47 (m, $1 \mathrm{H}), 7.65(\mathrm{~d}, J=7.6 \mathrm{~Hz}, 1 \mathrm{H}) ;{ }^{13} \mathrm{C} \mathrm{NMR}\left(100 \mathrm{MHz}, \mathrm{CDCl}_{3}\right) \delta: 12.60$ (1 $\mathrm{CH}_{3}$ ), 28.38 (1 $\mathrm{CH}_{2}$ ), 120.90 (1 quat. C), 122.64 (1 quat. C), $127.18(1 \mathrm{CH}), 128.35(3 \mathrm{CH}), 128.72(1 \mathrm{CH}), 128.84(2 \mathrm{CH})$, 130.27 (1 CH), 132.82 (1 CH), 140.13 (1 quat. C), 142.65 (1 quat. C), 143.53 (1 quat. C); HRMS (ESI, $m / z$ ) calcd. for $\mathrm{C}_{16} \mathrm{H}_{15} \mathrm{Br}_{2}[\mathrm{M}+\mathrm{H}]^{+}:$364.9535; Found 364.9545.

(Z)-1-Bromo-2-(5-bromooct-4-en-4-yl)-4-fluorobenzene (1e). Yellow oil, isolated yield 91\% (662 mg); ${ }^{1} \mathrm{H}$ NMR (400 MHz, $\left.\mathrm{CDCl}_{3}\right) \delta: 0.90(\mathrm{t}, J=7.2 \mathrm{~Hz}, 3 \mathrm{H}), 1.01(\mathrm{t}, J=7.4 \mathrm{~Hz}, 3 \mathrm{H})$, 1.23-1.43 (m, 2H), 1.64-1.77 (m, 2H), 2.23-2.49 (m, 2H), 2.51-2.74 (m, 2H), 6.80 (dd, $J=8.9,3.0 \mathrm{~Hz}, 1 \mathrm{H}), 7.01(\mathrm{dd}, J=7.7$, $2.2 \mathrm{~Hz}, 1 \mathrm{H}), 7.51(\mathrm{~d}, J=8.8 \mathrm{~Hz}, 1 \mathrm{H}) ;{ }^{13} \mathrm{C} \mathrm{NMR}\left(100 \mathrm{MHz}, \mathrm{CDCl}_{3}\right)$ $\delta: 13.18\left(1 \mathrm{CH}_{3}\right), 13.96\left(1 \mathrm{CH}_{3}\right), 21.03\left(1 \mathrm{CH}_{2}\right), 21.65\left(1 \mathrm{CH}_{2}\right)$, 36.00 ( $\left.1 \mathrm{CH}_{2}\right), 38.66\left(1 \mathrm{CH}_{2}\right), 114.28(\mathrm{~d}, J=21.0 \mathrm{~Hz}, 1 \mathrm{CH})$, $119.84(\mathrm{~d}, J=24.3 \mathrm{~Hz}, 1 \mathrm{CH}), 122.60$ (d, $J=9.5 \mathrm{~Hz}, 1$ quat. $\mathrm{C}$ ), 127.48 (1 quat. C), 131.31 (d, $J=8.3 \mathrm{~Hz}, 1 \mathrm{CH}$ ), 139.18 (d, $J=1.2$ Hz, 1 quat. C), 145.73 (d, $J=8.0 \mathrm{~Hz}, 1$ quat. C), 161.16 (d, $J=$ $248.5 \mathrm{~Hz}, 1$ quat. C); HRMS (EI, $m / z$ ) calcd. for $\mathrm{C}_{14} \mathrm{H}_{17} \mathrm{Br}_{2} \mathrm{~F}$ [M]: 363.9661; Found 363.9659.

(Z)-1-Bromo-2-(5-bromooct-4-en-4-yl)-4-methylbenzene (1f). Colorless oil, isolated yield 91\% (652 mg); ${ }^{1} \mathrm{H}$ NMR (400 MHz, $\left.\mathrm{CDCl}_{3}\right) \delta: 0.89(\mathrm{t}, J=7.2 \mathrm{~Hz}, 3 \mathrm{H}), 1.02(\mathrm{t}, J=7.2 \mathrm{~Hz}, 3 \mathrm{H})$, 1.26-1.44 (m, 2H), 1.65-1.76 (m, 2H), 2.24-2.31 (m, 4H, $\left.\mathrm{CH}_{3}+\mathrm{CH}_{2}\right), 2.42-2.50(\mathrm{~m}, 1 \mathrm{H}), 2.53-2.73(\mathrm{~m}, 2 \mathrm{H}), 6.93(\mathrm{~d}, J=7.8$ 
$\mathrm{Hz}, 1 \mathrm{H}), 7.08$ (d, $J=8.6 \mathrm{~Hz}, 1 \mathrm{H}), 7.41(\mathrm{~s}, 1 \mathrm{H}) ;{ }^{13} \mathrm{C}$ NMR $(100$ $\left.\mathrm{MHz}, \mathrm{CDCl}_{3}\right) \delta: 13.19\left(1 \mathrm{CH}_{3}\right), 13.99\left(1 \mathrm{CH}_{3}\right), 20.77\left(1 \mathrm{CH}_{3}\right)$, 21.05 (1 CH 2$), 21.68\left(1 \mathrm{CH}_{2}\right), 36.11\left(1 \mathrm{CH}_{2}\right), 38.68\left(1 \mathrm{CH}_{2}\right)$, 122.17 (1 quat. C), 126.74 (1 quat. C), 127.83 (1 CH), 130.12 (1 $\mathrm{CH}), 133.07$ (1 CH), 138.38 (1 quat. C), 139.89 (1 quat. C), 141.12 (1 quat. C); HRMS (ESI, $m / z$ ) calcd. for $\mathrm{C}_{15} \mathrm{H}_{21} \mathrm{Br}_{2}$ $[\mathrm{M}+\mathrm{H}]+$ : 359.0005; Found 359.0009.

(Z)-1-Bromo-2-(5-bromooct-4-en-4-yl)-4-methoxybenzene (1g). Colorless oil, isolated yield 93\% (710 mg); ${ }^{1} \mathrm{H}$ NMR (400 $\left.\mathrm{MHz} \mathrm{CDCl}_{3}\right) \delta: 0.90(\mathrm{t}, J=7.3 \mathrm{~Hz}, 3 \mathrm{H}), 1.01(\mathrm{t}, J=7.4 \mathrm{~Hz}, 3 \mathrm{H})$, 1.26-1.48 (m, 2H), 1.67-1.76 (m, 2H), 2.24-2.48 (m, 2H), 2.51-2.73 (m, 2H), $3.79(\mathrm{~s}, 3 \mathrm{H}), 6.84(\mathrm{~d}, J=8.5,1 \mathrm{H}), 6.96(\mathrm{~d}, J=$ $8.5 \mathrm{~Hz}, 1 \mathrm{H}), 7.13(\mathrm{~s}, 1 \mathrm{H}) ;{ }^{13} \mathrm{C} \mathrm{NMR}\left(100 \mathrm{MHz}, \mathrm{CDCl}_{3}\right) \delta: 13.19(1$ $\left.\mathrm{CH}_{3}\right), 13.99\left(1 \mathrm{CH}_{3}\right), 21.05\left(1 \mathrm{CH}_{2}\right), 21.69\left(1 \mathrm{CH}_{2}\right), 36.22\left(1 \mathrm{CH}_{2}\right)$, 38.73 (1 CH$), 55.39\left(1 \mathrm{CH}_{3}\right), 113.24(1 \mathrm{CH}), 117.61(1 \mathrm{CH})$, 122.71 (1 quat. C), 127.23 (1 quat. C), 130.85 (1 CH), 136.50 (1 quat. C), 139.66 (1 quat. C), 158.81 ( 1 quat. C); HRMS (ESI, $m / z$ ) calcd. for $\mathrm{C}_{15} \mathrm{H}_{21} \mathrm{Br}_{2} \mathrm{O}[\mathrm{M}+\mathrm{H}]+$ : 374.9954; Found 374.9965.

\subsection{Analytical data for the COT derivatives}

(5Z,11Z)-5,6,11,12-Tetrapropyldibenzo[a,e]cyclooctatetraene (3a) [19]. Yellow oil, isolated yield 83\% (93 mg); ${ }^{1} \mathrm{H}$ NMR (400 $\left.\mathrm{MHz}_{\mathrm{CDCl}}\right) \delta: 0.95(\mathrm{t}, J=7.6 \mathrm{~Hz}, 12 \mathrm{H}), 1.42-1.51(\mathrm{~m}, 8 \mathrm{H})$, 2.35-2.43 (m, 8H), 6.91-6.97 (m, 8H); ${ }^{13} \mathrm{C}$ NMR (100 MHz, $\left.\mathrm{CDCl}_{3}\right) \delta: 14.66\left(4 \mathrm{CH}_{3}\right), 22.43\left(4 \mathrm{CH}_{2}\right), 36.68\left(4 \mathrm{CH}_{2}\right), 125.40(4$ $\mathrm{CH}), 126.63$ (4 CH), 137.97 (4 quat. C), 143.23 (4 quat. C).

(5Z,11Z)-5,6,11,12-Tetraethyldibenzo[a,e]cyclooctatetraene (3b) [19]. Yellow oil, isolated yield 74\% (70 mg); ${ }^{1} \mathrm{H}$ NMR (400 $\left.\mathrm{MHz}, \mathrm{CDCl}_{3}\right) \delta: 1.06(\mathrm{t}, J=7.6 \mathrm{~Hz}, 12 \mathrm{H}), 2.41-2.54(\mathrm{~m}, 8 \mathrm{H})$, 6.94-6.99 (m, 8H); ${ }^{13} \mathrm{C} \mathrm{NMR}\left(100 \mathrm{MHz}, \mathrm{CDCl}_{3}\right) \delta: 14.03\left(4 \mathrm{CH}_{3}\right)$, $27.15\left(4 \mathrm{CH}_{2}\right), 125.46(4 \mathrm{CH}), 126.65(4 \mathrm{CH}), 138.72$ (4 quat. C), 143.04 (4 quat. C).

(5Z,11Z)-5,6,11,12-Tetramethyldibenzo[a,e]cyclooctatetraene (3c). White solid, isolated yield 85\% (66 mg); m.p: 155.4-157.1 ${ }^{\circ} \mathrm{C}$; ${ }^{1} \mathrm{H}$ NMR (400 MHz, $\left.\mathrm{CDCl}_{3}\right) \delta: 2.05(\mathrm{~s}, 12 \mathrm{H}), 6.95-7.01(\mathrm{~m}$, $8 \mathrm{H}) ;{ }^{13} \mathrm{C} \mathrm{NMR}\left(100 \mathrm{MHz}, \mathrm{CDCl}_{3}\right) \delta: 21.03\left(4 \mathrm{CH}_{3}\right), 125.77(4 \mathrm{CH})$, 126.76 (4 CH), 132.51 (4 quat. C), 143.52 (4 quat. C); HRMS (ESI, $m / z$ ) calcd. for $\mathrm{C}_{20} \mathrm{H}_{21}[\mathrm{M}+\mathrm{H}]^{+}:$261.1638; Found 261.1641.

(5Z,11Z)-5,11-Diethyl-6,12-diphenyldibenzo[a,e]cyclooctatetraene (3d). Yellow oil, isolated yield $66 \%$ (81 mg); ${ }^{1} \mathrm{H}$ NMR (400 MHz, $\left.\mathrm{CDCl}_{3}\right) \delta: 0.41(\mathrm{t}, J=7.5 \mathrm{~Hz}, 6 \mathrm{H}), 2.16-2.25(\mathrm{~m}, 4 \mathrm{H})$, 6.78-6.80 (m, 4H), 7.10-7.13 (m, 4H), 7.28-7.44 (m, 10H); ${ }^{13} \mathrm{C}$ NMR (125 MHz, $\left.\mathrm{CDCl}_{3}\right) \delta: 12.53\left(2 \mathrm{CH}_{3}\right), 27.91\left(2 \mathrm{CH}_{2}\right), 126.21$ (2 CH), $126.24(2 \mathrm{CH}), 126.65(2 \mathrm{CH}), 126.80(2 \mathrm{CH}), 127.55(4$ $\mathrm{CH}), 128.66$ (2 CH), 129.18 (4 CH), 139.41 (2 quat. C), 140.20 (4 quat. C), 142.53 (2 quat. C), 142.63 (2 quat. C); HRMS (ESI, $m / z$ ) calcd. for $\mathrm{C}_{32} \mathrm{H}_{29}[\mathrm{M}+\mathrm{H}]^{+}$: 413.2264; Found 413.2271.

(5Z,11Z)-2,8-Difluoro-5,6,11,12-tetrapropyldibenzo[a,e]cyclooctatetraene (3e). Colorless oil, isolated yield 66\% (81 mg); ${ }^{1} \mathrm{H}$ NMR (400 MHz, $\left.\mathrm{CDCl}_{3}\right) \delta$ : 0.93-0.98 (m, 12H), 1.39-1.48 (m, $8 \mathrm{H}), 2.33-2.37(\mathrm{~m}, 8 \mathrm{H}), 6.61-6.71(\mathrm{~m}, 4 \mathrm{H}), 6.86-6.90(\mathrm{~m}, 2 \mathrm{H})$; ${ }^{13} \mathrm{C}$ NMR (125 MHz, $\left.\mathrm{CDCl}_{3}\right) \delta: 14.59\left(4 \mathrm{CH}_{3}\right), 22.31\left(2 \mathrm{CH}_{2}\right)$, 22.40 (2 $\mathrm{CH}_{2}$ ), $36.67\left(4 \mathrm{CH}_{2}\right), 112.72$ (d, $J=21.1 \mathrm{~Hz}, 2 \mathrm{CH}$ ), 113.07 (d, $J=20.4 \mathrm{~Hz}, 2 \mathrm{CH}), 128.17$ (d, $J=8.2 \mathrm{~Hz}, 2 \mathrm{CH}$ ), 137.63 (d, $J=1.5 \mathrm{~Hz}, 2$ quat. C), 137.91 (2 quat. C), 138.76 (d, $J=3.0 \mathrm{~Hz}$, 2 quat. C), 145.06 (d, $J=7.2 \mathrm{~Hz}, 2$ quat. C), 160.73 (d, $J=242.8$
$\mathrm{Hz}, 2$ quat. C); HRMS (ESI, $m / z$ ) calcd. for $\mathrm{C}_{28} \mathrm{H}_{35} \mathrm{~F}_{2}[\mathrm{M}+\mathrm{H}]^{+}$: 409.2701; Found 409.2707.

(5Z,11Z)-2,8-Dimethyl-5,6,11,12-tetrapropyldibenzo[a,e]cyclooctatetraene (3f). Yellow oil, isolated yield 70\% $(84 \mathrm{mg}) ;{ }^{1} \mathrm{H}$ NMR (400 MHz, $\left.\mathrm{CDCl}_{3}\right) \delta:$ 0.92-0.97 (m, $\left.12 \mathrm{H}\right), 1.40-1.49(\mathrm{~m}$, $8 \mathrm{H}), 2.17(\mathrm{~s}, 6 \mathrm{H}), 2.32-2.36(\mathrm{~m}, 8 \mathrm{H}), 6.75-6.84(\mathrm{~m}, 6 \mathrm{H}) ;{ }^{13} \mathrm{C}$ NMR (125 MHz, $\left.\mathrm{CDCl}_{3}\right) \delta: 14.68\left(4 \mathrm{CH}_{3}\right), 21.12\left(2 \mathrm{CH}_{3}\right), 22.52$ (4 $\mathrm{CH}_{2}$ ), $36.86\left(4 \mathrm{CH}_{2}\right), 126.27(2 \mathrm{CH}), 126.44(2 \mathrm{CH}), 127.26$ (2 $\mathrm{CH}$ ), 134.48 (2 quat. C), 137.71 (2 quat. C), 137.87 (2 quat. C), 140.46 (2 quat. C), 143.21 (2 quat. C); HRMS (ESI, $m / z$ ) calcd. for $\mathrm{C}_{30} \mathrm{H}_{41}[\mathrm{M}+\mathrm{H}]+$ : 401.3203; Found 401.3205.

(5Z,11Z)-2,8-Dimethoxy-5,6,11,12-tetrapropyldibenzo[a,e]cyclooctatetraene (3g). Yellow oil, isolated yield 73\% (95 mg); ${ }^{1} \mathrm{H}$ NMR (400 MHz, $\left.\mathrm{CDCl}_{3}\right) \delta:$ 0.92-0.96 (m, $\left.12 \mathrm{H}\right), 1.41-1.49(\mathrm{~m}$, $8 \mathrm{H}), 2.31-2.38(\mathrm{~m}, 8 \mathrm{H}), 3.69(\mathrm{~s}, 6 \mathrm{H}), 6.48(\mathrm{~s}, 2 \mathrm{H}), 6.55(\mathrm{~d}, J=8.5$ $\mathrm{Hz}, 2 \mathrm{H}), 6.85$ (d, $J=8.5 \mathrm{~Hz}, 2 \mathrm{H}) ;{ }^{13} \mathrm{C} \mathrm{NMR}\left(125 \mathrm{MHz}, \mathrm{CDCl}_{3}\right) \delta$ : $14.65\left(4 \mathrm{CH}_{3}\right), 22.47\left(2 \mathrm{CH}_{2}\right), 22.57\left(2 \mathrm{CH}_{2}\right), 36.88\left(4 \mathrm{CH}_{2}\right)$, $54.93\left(2 \mathrm{CH}_{3}\right), 111.07(2 \mathrm{CH}), 111.99(2 \mathrm{CH}), 127.50(2 \mathrm{CH})$, 136.01 (2 quat. C), 137.67 (2 quat. C), 137.85 (2 quat. C), 144.50 (2 quat. C), 156.98 (2 quat. C); HRMS (ESI, $m / z$ ) calcd. for $\mathrm{C}_{30} \mathrm{H}_{41} \mathrm{O}_{2}[\mathrm{M}+\mathrm{H}]^{+}:$433.3101; Found 433.3092.

Tetraphenylene (3h). White solid, isolated yield 74\% (41 mg) [58]; ${ }^{1} \mathrm{H}$ NMR (400 MHz, $\left.\mathrm{CDCl}_{3}\right) \delta: 7.13-7.17(\mathrm{~m}, 8 \mathrm{H})$, 7.25-7.28 (m, 8H); $\left.{ }^{13} \mathrm{C} \mathrm{NMR} \mathrm{(125} \mathrm{MHz,} \mathrm{CDCl}_{3}\right) \delta: 127.22(8 \mathrm{CH})$, $129.01(8 \mathrm{CH}), 141.55$ (8 quat. C).

(1Z,3Z,5Z,7Z)-1,2,3,4,5,6,7,8-Octaethylcycloocta-1,3,5,7-tetraene (3i) [20]. Colorless oil, isolated yield 61\% (60 mg); ${ }^{1} \mathrm{H}$ NMR (400 MHz, $\left.\mathrm{CDCl}_{3}\right) \delta: 0.94(\mathrm{t}, J=7.5,24 \mathrm{H}), 1.97-2.11(\mathrm{~m}, 16 \mathrm{H})$; ${ }^{13} \mathrm{C}$ NMR (100 MHz, $\left.\mathrm{CDCl}_{3}\right) \delta: 14.22\left(8 \mathrm{CH}_{3}\right), 23.54\left(8 \mathrm{CH}_{2}\right)$, 138.32 (8 quat. C).

\subsection{X-ray crystallographic studies of $3 c$}

Single crystals of 3c suitable for X-ray analysis were grown in solution of $\mathrm{CDCl}_{3}$. Data collection for 3c was performed at $-93{ }^{\circ} \mathrm{C}$ on a Rigaku CCD SATURN724 diffractometer using graphite-monochromated Mo $K_{\alpha}$ radiation $(\lambda=0.71073 \AA)$. The structures were solved using the SHELXTL program [59] or with the XS [60] structure solution program using Direct Methods and refined with the olex2.refine refinement package using Gauss-Newton minimization $[60,61]$. These data can be obtained free of charge from the Cambridge Crystallographic Data Centre via www.ccdc.cam.ac.uk/data_request/cif. CCDC 1006078 (3c).

\section{Results and discussion}

\subsection{Optimization of reaction conditions}

Initially, we investigated the reaction of 1a, which afforded the dibenzo[a,e]cyclooctatetraene derivative 3a. Various reaction conditions were screened (Table 1). We found that the reaction was very sensitive to the choice of base [62]. LiOEt was found to be the most effective base for this reaction, while stronger bases (e.g. $\mathrm{NaOt}$ - $\mathrm{Bu}$ and $\mathrm{KO} t-\mathrm{Bu}$ ) resulted in the decomposition of $\mathbf{1 a}$ and weak bases (e.g. NaOAc) afforded no product at all (entries 3-5). The amount of the base used in this 
Table 1

Optimization of reaction conditions.

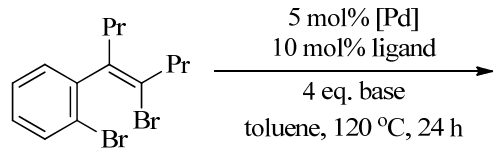

1a

\begin{tabular}{|c|c|c|c|c|}
\hline Entry & {$[\mathrm{Pd}]$} & Ligand & Base & Yield of $\mathbf{3} \mathbf{a}^{\mathrm{a}}(\%)$ \\
\hline 1 & $\mathrm{PdCl}_{2}(\mathrm{PhCN})_{2}$ & $t-\mathrm{Bu}_{3} \mathrm{P}$ & $\mathrm{LiO} t-\mathrm{Bu}$ & trace \\
\hline 2 & $\mathrm{PdCl}_{2}\left(\mathrm{PPh}_{3}\right)_{2}$ & $t-\mathrm{Bu}_{3} \mathrm{P}$ & $\mathrm{LiO} t-\mathrm{Bu}$ & trace \\
\hline 3 & $\mathrm{PdCl}_{2}\left(\mathrm{PPh}_{3}\right)_{2}$ & $t-\mathrm{Bu}_{3} \mathrm{P}$ & $\mathrm{NaO} t-\mathrm{Bu}$ & trace \\
\hline 4 & $\mathrm{PdCl}_{2}\left(\mathrm{PPh}_{3}\right)_{2}$ & $t-\mathrm{Bu}_{3} \mathrm{P}$ & $\mathrm{KO} t-\mathrm{Bu}$ & $-\mathrm{b}$ \\
\hline 5 & $\mathrm{PdCl}_{2}\left(\mathrm{PPh}_{3}\right)_{2}$ & $t-\mathrm{Bu}_{3} \mathrm{P}$ & $\mathrm{NaOAc}$ & $-\mathrm{b}$ \\
\hline 6 & $\mathrm{PdCl}_{2}\left(\mathrm{PPh}_{3}\right)_{2}$ & $t-\mathrm{Bu}_{3} \mathrm{P}$ & $\mathrm{Cs}_{2} \mathrm{CO}_{3} \mathrm{c}$ & 10 \\
\hline 7 & $\mathrm{PdCl}_{2}\left(\mathrm{PPh}_{3}\right)_{2}$ & $t-\mathrm{Bu}_{3} \mathrm{P}$ & LiOEt & 85 \\
\hline 8 & $\mathrm{PdCl}_{2}\left(\mathrm{PPh}_{3}\right)_{2}$ & $\mathrm{P}(2 \text {-furan })_{3}$ & LiOEt & 67 \\
\hline 9 & $\mathrm{PdCl}_{2}\left(\mathrm{PPh}_{3}\right)_{2}$ & Sphos & LiOEt & 53 \\
\hline 10 & $\mathrm{PdCl}_{2}\left(\mathrm{PPh}_{3}\right)_{2}$ & $\mathrm{PPh}_{3}$ & LiOEt & 42 \\
\hline 11 & $\operatorname{Pd}\left(t-\mathrm{Bu}_{3} \mathrm{P}\right)_{2}$ & $t-\mathrm{Bu}_{3} \mathrm{P}$ & LiOEt & $89(83)$ \\
\hline 12 & $\operatorname{Pd}\left(t-\mathrm{Bu}_{3} \mathrm{P}\right)_{2}$ & - & LiOEt & 41 \\
\hline 13 & $\operatorname{Pd}\left(t-\mathrm{Bu}_{3} \mathrm{P}\right)_{2}$ & - & $\mathrm{LiO} t-\mathrm{Bu}$ & trace \\
\hline 14 & $\operatorname{Pd}\left(t-\mathrm{Bu}_{3} \mathrm{P}\right)_{2}$ & - & $\mathrm{LiO} t-\mathrm{Bu}^{\mathrm{c}}$ & 47 \\
\hline 15 & $\operatorname{Pd}\left(t-\mathrm{Bu}_{3} \mathrm{P}\right)_{2}$ & $t-\mathrm{Bu}_{3} \mathrm{P}$ & LiOEt d & trace \\
\hline 16 & $\operatorname{Pd}\left(t-\mathrm{Bu}_{3} \mathrm{P}\right)_{2}$ & $t-\mathrm{Bu}_{3} \mathrm{P}$ & LiOEt ${ }^{\mathrm{e}}$ & 11 \\
\hline 17 & $\operatorname{Pd}\left(t-\mathrm{Bu}_{3} \mathrm{P}\right)_{2}$ & $t-\mathrm{Bu}_{3} \mathrm{P}$ & LiOEt ${ }^{f}$ & 35 \\
\hline 18 & $\operatorname{Pd}\left(t-\mathrm{Bu}_{3} \mathrm{P}\right)_{2}$ & $t-\mathrm{Bu}_{3} \mathrm{P}$ & LiOEt $\mathrm{g}$ & 70 \\
\hline
\end{tabular}

${ }^{a}$ GC yield $\left(n-\mathrm{C}_{12} \mathrm{H}_{26}\right.$ as internal standard), isolated yield in parenthesis. b No product was observed.

c 4 eq. of $i$-PrOH was added.

d 0.5 eq. of LiOEt was used.

e 1.0 eq. of LiOEt was used.

f 2.0 eq. of LiOEt was used.

g 3.0 eq. of LiOEt was used.

reaction strongly affected the yield of product 3a (entries 11, 15-18). The optimum conditions for this transformation were found to be $\mathrm{Pd}\left(t-\mathrm{Bu}_{3} \mathrm{P}\right)_{2}$ (5 mol\%), $t$-Bu $3 \mathrm{P}_{3} \mathrm{P}(10 \mathrm{~mol} \%)$, LiOEt (4 equiv), toluene, $120{ }^{\circ} \mathrm{C}, 24 \mathrm{~h}$. Under these optimized reaction conditions, the product 3a was obtained in $83 \%$ isolated yield.

\subsection{Reaction scope}

With the above optimized reaction conditions in hand, we investigated the scope of this reaction. As shown in Scheme 2, dibenzo[a,e]cyclooctatetraene derivatives $\mathbf{3 a - 3 h}$ could be obtained in moderate to good yields. The substituents in the vinyl group played an important role in this reaction. Those substrates substituted with aliphatic groups reacted smoothly and 3a-3c were generated in good yields. Changing the aliphatic group to an aromatic one caused a slight decrease in the yield (3d). A phenyl ring substituted with different groups generally afforded moderate to good yields of the corresponding products (3e-3g). Those substituted with electron-donating groups gave the products in slightly higher yields than those with the electron-withdrawing ones. The tetraphenylene product $\mathbf{3 h}$ could also be obtained in this reaction. It is noteworthy that this coupling reaction proceeded highly selectively. The stereochemistry of compound 3c was determined by its single crystal X-ray structural analysis (Fig. 1).

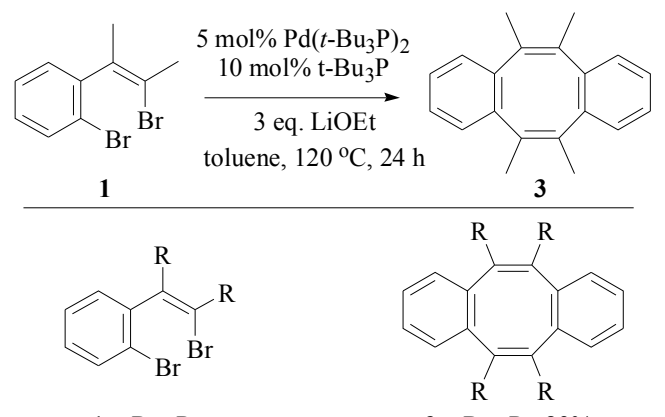

1a: $R=\operatorname{Pr}$

1b: $\mathrm{R}=\mathrm{Et}$

1c: $\mathrm{R}=\mathrm{Me}$

3a: $\mathrm{R}=\operatorname{Pr}, 83 \%$

3b: $\mathrm{R}=\mathrm{Et}, 74 \%$

3c: $\mathrm{R}=\mathrm{Me}, 85 \%$<smiles>CC/C(=C(\Br)c1ccccc1)c1ccccc1Br</smiles>

Et $\mathrm{Ph}$

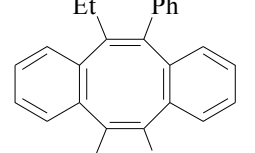

1d<smiles>[R]c1ccc(Br)c(C(CCC)=C(Br)Br)c1</smiles>

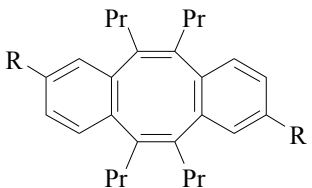

1e: $R=F$
1f: $R=M e$
1g: $R=O M e$

3e: $\mathrm{R}=\mathrm{F}, 66 \%$

3f: $\mathrm{R}=\mathrm{Me}, 70 \%$

3g: $\mathrm{R}=\mathrm{OMe}, 73 \%$

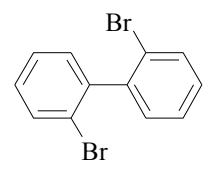

$1 \mathbf{h}$

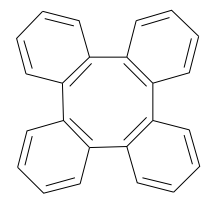

3h: $45 \%$

Scheme 2. Formation of dibenzo[a,e]cyclooctatetraenes $\mathbf{3}$ via intramolecular homo-coupling of $o$-bromo-(2-bromovinyl)benzenes $\mathbf{1}$.

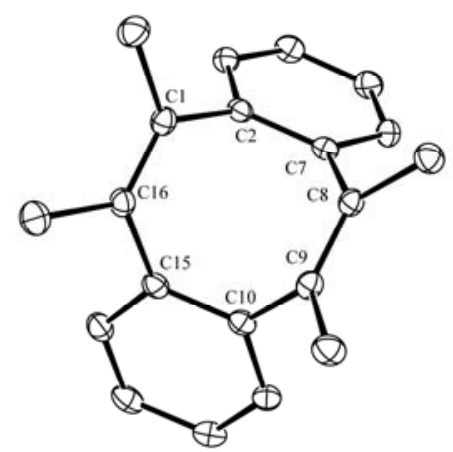

Fig. 1. ORTEP drawing of 3c with 30\% thermal ellipsoids. Hydrogen atoms omitted for clarity.

Meanwhile, the 1,2,3,4-tetraethyl-1,4-dibromo-1,3-butadiene 2 could also be reacted to afford the corresponding octaethylcyclooctatetraene $\mathbf{3 i}$ (Scheme 3).

\subsection{Reaction mechanism}

To gain insight into this reaction, several experiments were carried out. (1) As shown in Table 1, only a trace amount of 3a 
<smiles>CCC(Br)=C(CC)C(CC)=C(Br)Br</smiles>

2

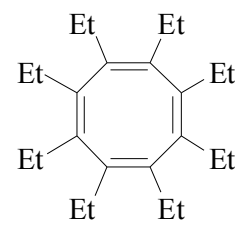

3i: $61 \%$
Scheme 3. Formation of octaethylcyclooctatetraene $3 \mathbf{i}$ via intramolecular homo-coupling of 1,2,3,4-tetraethyl-1,4-dibromo-1,3-butadiene 2 .

could be observed in the presence of 0.5 eq. LiOEt and mainly starting material 1a remained. Increasing the amount of LiOEt enhanced the yield of $\mathbf{1 a}$ (Table 1, entries 11 and 15-18). (2) Lemaire et al. $[63,64]$ reported that $i$-PrOH could be used as a reducing agent to regenerate the $\mathrm{Pd}(0)$ active species from Pd(II) species with the concomitant formation of acetone. In this COT formation reaction, both the gas and the liquid phases of the reaction were analyzed using GC/MS analysis. The examination of the reaction mixture showed that small amounts of acetaldehyde and ethyl acetate were formed in this process [65]. (3) The addition of a reductant (e.g $i$-PrOH) was essential when LiOt-Bu was used instead of LiOEt in this reaction (Table 1, entries 13 and 14).

Based on the above preliminary results, a plausible catalytic cycle for the formation of cyclooctatetraene is shown in Scheme 4 [57,66-70]. Intermediate 5 would be generated via oxidative addition of $\mathbf{1}$ to the $\operatorname{Pd}(0)$ species. Then ligand exchange and a subsequent intramolecular migratory insertion would lead to the formation of palladacycle intermediate $\mathbf{6}$. This palladacycle could undergo a second oxidative addition with another molecule of $\mathbf{1}$ to generate intermediate $\mathbf{7}$ and a subsequent reductive elimination would give intermediate 8. A second ligand exchange followed by intramolecular migratory insertion would lead to the formation of the 9-membered palladacycle intermediate 9. Finally, reductive elimination of $\mathbf{9}$ would then release the cyclooctatetraene 3 and regenerate the active $\operatorname{Pd}(0)$ species to re-enter the catalytic cycle.

As reported in our previous work [57], dibenzo[a,c]cyclooctatetraenes could be selectively generated via a Pd-catalyzed homo-coupling of borylvinyl iodobenzene derivatives (Scheme 5, Eq. 1). A control experiment was carried out to investigate the different selectivity of these two reactions. When 1a was treated with $\mathrm{PdCl}_{2}\left(\mathrm{PPh}_{3}\right)_{2}$, $i$-PrOH and $\mathrm{Cs}_{2} \mathrm{CO}_{3}$, formation of trace amounts of both $\mathbf{3 a}$ and $\mathbf{3} \mathbf{a}^{\prime}$ could be observed, along with the remaining starting material 1a (Scheme 5, Eq. 2 ). The addition of $10 \mathrm{mmol} \%$ of $t-\mathrm{Bu}_{3} \mathrm{P}$ led to trace amounts of 3a' and $10 \%$ of $\mathbf{3 a}$ with remaining $\mathbf{1 a}$ (Table 1, entry 6). The different selectivity could be explained by the steric effects of the ligand. When $\mathrm{Cs}_{2} \mathrm{CO}_{3}$ is added to the reaction, it can act as a carboxylate ligand, coordinating to the Pd(IV) center. The corresponding Pd(IV) intermediate $\mathbf{7}$ ' would therefore have a different configuration to intermediate $\mathbf{7}$, and thus result in different selectivity in the subsequent reductive elimination (Scheme 5).

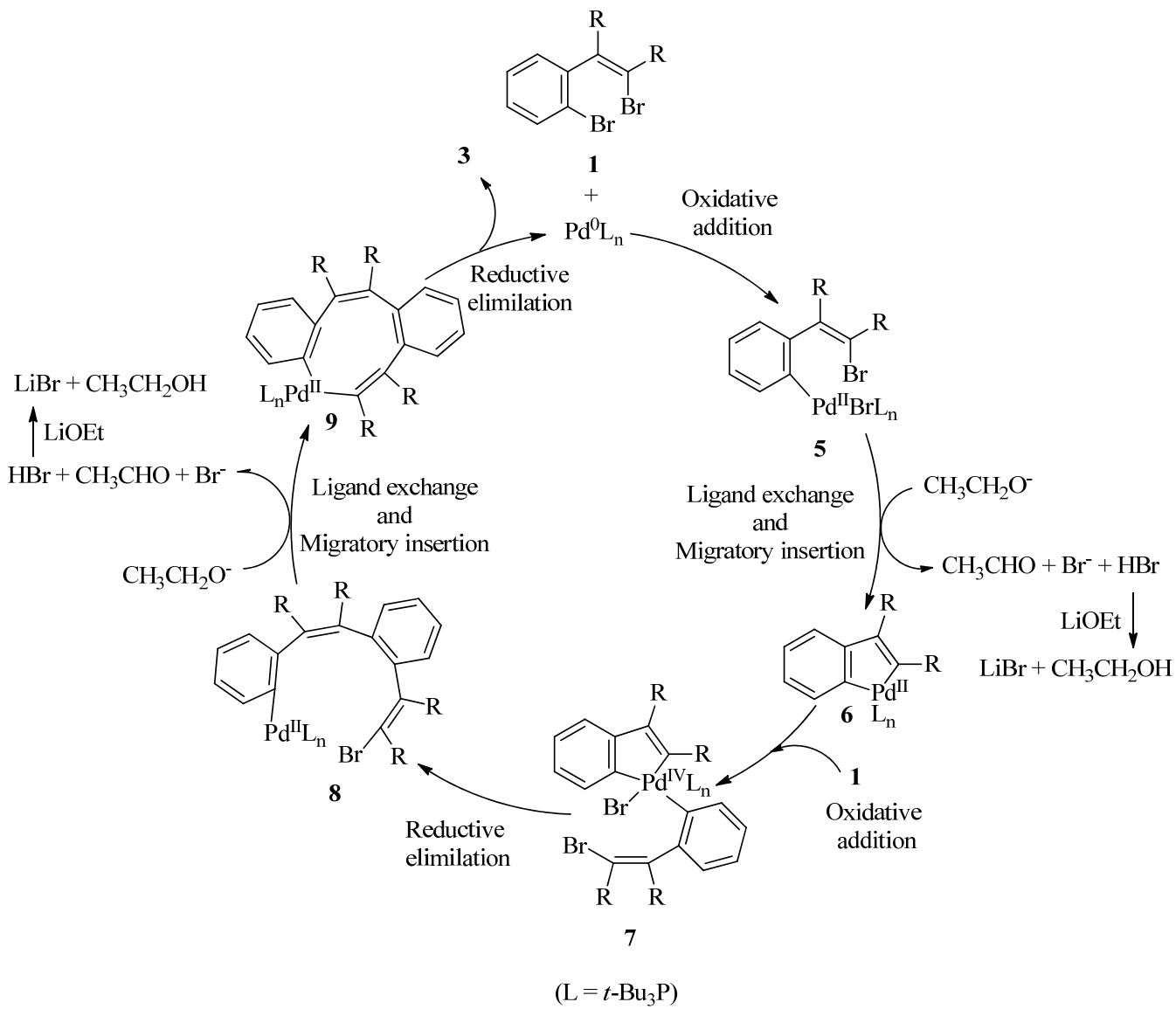

Scheme 4. A proposed mechanism of the formation of cyclooctatetraenes. 


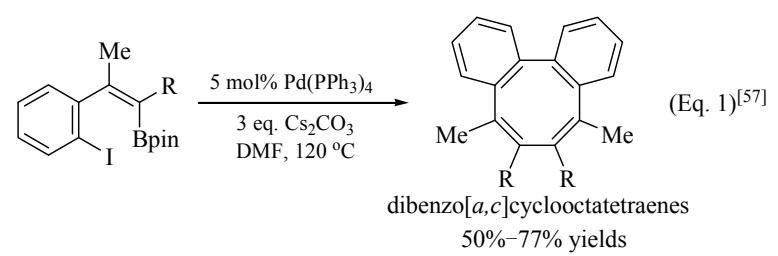

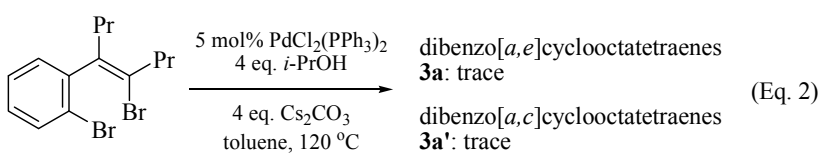

$1 \mathrm{a}$

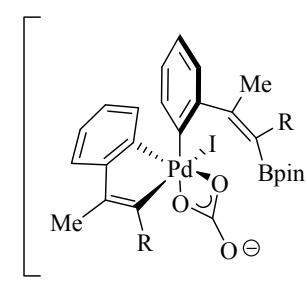

$7^{\prime}$

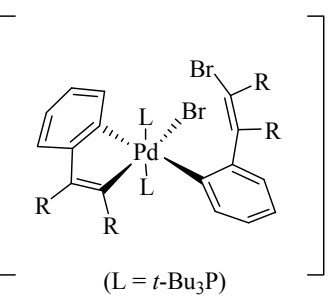

7
Scheme 5. Steric effects of ligands in the regioselective generation of cyclooctatetraenes.

\section{Conclusions}

We have developed an efficient Pd-catalyzed intramolecular homo-coupling reaction of dibromo-substituted compounds to synthesize multi-substituted cyclooctatetraenes, in particular dibenzo[a,e]cyclooctatetraene derivatives. We propose that the steric effects of the ligands control the regioselectivity of the formation of the dibenzocyclooctatetraenes.

\section{References}

[1] Huisgen R, Mietzsch F. Angew Chem Int Ed, 1964, 3: 83

[2] Paquette L A. Tetrahedron, 1975, 31: 2855

[3] Schumann H. Angew Chem Int Ed, 1984, 23: 474

[4] Paquette L A. Acc Chem Res, 1993, 26: 57

[5] Klärner F G. Angew Chem Int Ed, 2001, 40: 3977

[6] Nishinaga T, Ohmae T, Iyoda M. Symmetry, 2010, 2: 76

[7] Edelmann F T. Angew Chem Int Ed, 1995, 34: 2466

[8] Edelmann F T. New JChem, 1995, 19: 535

[9] Edelmann F T. In: Abel E W, Stone F G A, Wilkinson G eds. Comprehensive Organometallic Chemistry II. Pergamon Press, Oxford, 1995

[10] Schumann H, Meese-Marktscheffel J A, Esser L. Chem Rev, 1995, 95: 865

[11] Wang C, Xi Z F. Chem Commun, 2007: 5119

[12] Komatsu K. Bull Chem Soc Jpn, 2001, 74: 407

[13] Hui C W, Mak T C W, Wong H N C. Tetrahedron, 2004, 60: 3523

[14] Lai C W, Lam C K, Lee H K, Mak T C W, Wong H N C. Org Lett, 2003, 5: 823

[15] Lawrie C J, Gable K P, Carpenter B K. Organometallics, 1989, 8: 2274

[16] Esser B, Bandyopadhyay A, Rominger F, Gleiter R. Chem Eur J, 2009, 15: 3368

[17] Carnes M, Buccella D, Decatur J, Steigerwald M L, Nuckolls C. Angew Chem Int Ed, 2008, 47: 2982
[18] Takahashi T, Sun W H, Nakajima K. Chem Commun, 1999: 1595

[19] Li G T, Fang H Y, Zhang S W, Xi Z F. Tetrahedron Lett, 2004, 45: 8399

[20] Wang C, Yuan J, Li G T, Wang Z T, Zhang S W, Xi Z F. J Am Chem Soc, 2006, 128: 4564

[21] Wei J N, Wang Z T, Zhang W X, Xi Z F. Org Lett, 2013, 15: 1222

[22] Geng W Z, Wei J N, Zhang W X, Xi Z F. J Am Chem Soc, 2014, 136: 610

[23] Yamamoto Y, Ohno T, Itoh K. Chem Commun, 1999: 1543

[24] Yamamoto Y, Ohno T, Itoh K. Chem Eur J, 2002, 8: 4734

[25] Chen C, Xi C J, Lai C B, Wang R J, Hong X Y. Eur J Org Chem, 2004: 647

[26] Wender P A, Christy J P. J Am Chem Soc, 2007, 129: 13402

[27] Wender P A, Christy J P, Lesser A B, Gieseler M T. Angew Chem Int $E d, 2009,48: 7687$

[28] Ubayama H, Sun W H, Takahashi T, Xi Z F. Chem Commun, 1998: 1931

[29] Eisch J J, Piotrowski A M, Han K I, Krüger C, Tsay Y H. Organometallics, 1985, 4: 224

[30] Edelbach B L, Lachicotte R J, Jones W D. J Am Chem Soc, 1998, 120: 2843

[31] Perthuisot C, Edelbach B L, Zubris D L, Simhai N, Iverson C N, Müller C, Satoh T, Jones W D. J Mol Catal A, 2002, 189: 157

[32] Masselot D, Charmant J P H, Gallagher T. J Am Chem Soc, 2006, 128: 694

[33] Schaub T, Backes M, Radius U. Organometallics, 2006, 25: 4196

[34] Ananikov V P, Hazipov O V, Beletskaya I P. Chem Asian J, 2011, 6: 306

[35] Negishi E, Cederbaum F E, Takahashi T. Tetrahedron Lett, 1986, 27: 2829

[36] Yasuike S, Ohta H, Shiratori S, Kurita J, Tsuchiya T. J Chem Soc, Chem Commun, 1993: 1817

[37] Teplý F, Stará I G, Starý I, Kollárovič A, Šaman D, Rulíšek L, Fiedler P. J Am Chem Soc, 2002, 124: 9175

[38] Barluenga J, Valdés C, Beltrán G, Escribano M, Aznar F. Angew Chem Int Ed, 2006, 45: 6893

[39] Ball C J, Gilmore J, Willis M C. Angew Chem Int Ed, 2012, 51: 5718

[40] Muzalevskiy V M, Nenajdenko V G, Shastin A V, Balenkova E S, Haufe G. Synthesis, 2009: 2249

[41] Corey J C, John C S, Ohmsted M C, Chang L S. J Organomet Chem, 1986, 304: 93

[42] Liu Y X, Stringfellow T C, Ballweg D, Guzei I A, West R. J Am Chem Soc, 2002, 124: 49

[43] Chang L S, Corey J Y. Organometallics, 1989, 8: 1885

[44] Shimizu M, Tomioka Y, Nagao I, Kadowaki T, Hiyama T. Chem Asian J, 2012, 7: 1644

[45] Willis M C, Brace G N, Findlay T J K, Holmes I P. Adv Synth Catal, 2006, 348: 851

[46] Shen Q L, Hartwig J F. J Am Chem Soc, 2006, 128: 10028

[47] Liang Y, Meng T H, Zhang H J, Xi, Z F. Synlett, 2011: 911

[48] Tadd A C, Matsuno A, Fielding M R, Willis M C. Org Lett, 2009, 11: 583

[49] Kundu D, Maity P, Ranu B C. Org Lett, 2014, 16: 1040

[50] Geng W Z, Zhang W X, Hao W, Xi Z F. J Am Chem Soc, 2012, 134: 20230

[51] Fang H Y, Li G T, Mao G L, Xi Z F. Chem EurJ, 2004, 10: 3444

[52] Liang Y, Geng W Z, Wei J N, Ouyang K B, Xi Z F. Org Biomol Chem, 2012, 10: 1537

[53] Xi Z F. Acc Chem Res, 2010, 43: 1342

[54] Wei J N, Liu L, Zhan M, Xu L, Zhang W X, Xi Z F. Angew Chem Int Ed, 2014, 53: 5634

[55] Li H, Wei B S, Xu L, Zhang W X, Xi Z F. Angew Chem Int Ed, 2013, 


\title{
Graphical Abstract
}

Chin. J. Catal., 2015, 36: 24-32 doi: 10.1016/S1872-2067(14)60201-1

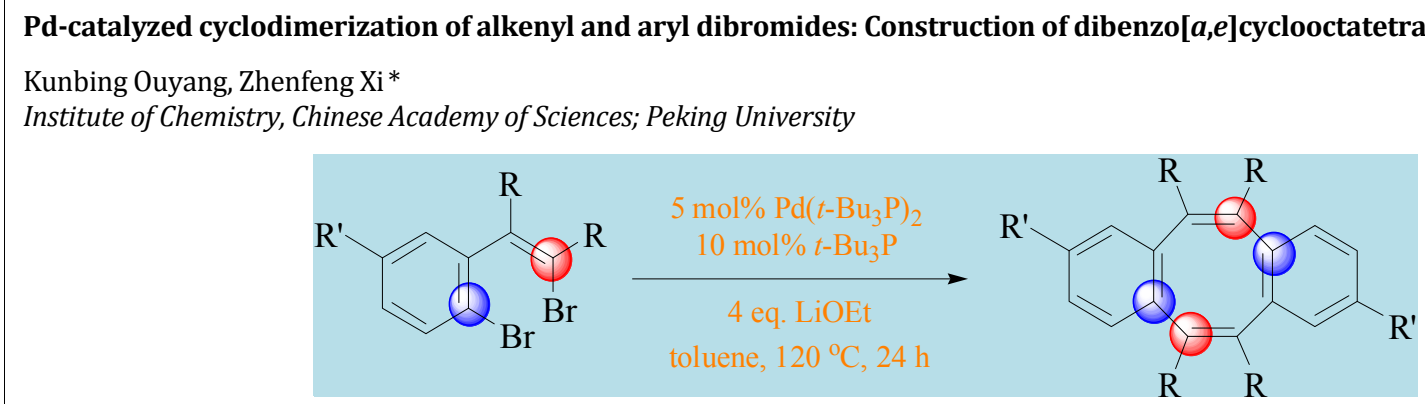

Dibenzo[a,e]cyclooctatetraene derivatives are efficiently and regioselectively generated from a palladium-catalyzed [4+4] cyclic homo-coupling of $o$-bromo-2-(2-bromovinyl)benzenes.

52: 10822

[56] Zhou Y, Zhang W X, Xi Z F. Organometallics, 2012, 31: 5546

[57] Zhang H J, Wei J N, Zhao F, Liang Y, Wang Z T, Xi Z F. Chem Commun, 2010, 46: 7439

[58] Schaub T, Radius U. Chem Eur J, 2005, 11: 5024

[59] Sheldrick G M. SHELXTL 5.10 for Windows NT: Structure Determination Software Programs. Bruker Analytical X-ray Systems, Inc., Madison, WI, 1997

[60] Sheldrick G M. Acta Cryst A, 2008, 64: 112

[61] Dolomanov O V, Bourhis L J, Gildea R J, Howard J A K, Puschmann H. J Appl Cryst, 2009, 42: 339

[62] Ouyang K B, Xi Z F. Acta Chim Sin, 2013, 71: 13

[63] Hassan J, Hathroubi C, Gozzi C, Lemaire M. Tetrahedron, 2001, 57:
7845

[64] Hassan J, Penalva V, Lavenot L, Gozzi C, Lemaire M. Tetrahedron, 1998, 54: 13793

[65] Shao L J, Du Y J, Zeng M F, Li X D, Shen W T, Zuo S F, Lu Y Q, Zhang X M, Qi C Z. Appl Organometal Chem, 2010, 24: 421

[66] Nadri S, Azadi E, Ataei A, Joshaghani M, Rafiee E. J Organomet Chem, 2011, 696: 2966

[67] Sehnal P, Taylor R J K, Fairlamb I J S. Chem Rev, 2010, 110: 824

[68] Xu L M, Li B J, Yang Z, Shi Z J. Chem Soc Rev, 2010, 39: 712

[69] Mu X, Wu T, Wang H Y, Guo Y L, Liu G S. J Am Chem Soc, 2012, 134: 878

[70] Canty A J, Patel J, Rodemann T, Ryan J H, Skelton B W, White A H. Organometallics, 2004, 23: 3466

\section{钯催化二溴代化合物自偶联环化合成二苯并 $[\boldsymbol{a}, \boldsymbol{e}]$ 环辛四烯}

\author{
欧阳昆冰 ${ }^{\mathrm{a}, \mathrm{b}}$, 席振峰 ${ }^{\mathrm{b}}$ * \\ a 北京分子科学国家实验室(筹), 中国科学院化学研究所, 北京 100190 \\ b 北京分子科学国家实验室(筹), 北京大学化学学院, 生物有机与分子工程教育部重点实验室, 北京100871
}

摘要: 由于独特的结构和广泛的应用, 多取代环辛四烯及其苯并稠环衍生物的合成方法研究具有重要意义. 本文报道了一种钯催 化烯基溴化物与芳基溴化物的偶联反应. 利用此钯催化的环化自偶联反应, 以中等至较好的收率高选择性地从双溴代芳基或烯 基化合物合成了多种二苯并 $[a, e]$ 环辛四烯衍生物.

关键词: 二溴代化合物; 环辛四烯; 二苯并 $[a, e]$ 环辛四烯; 1,3 -丁二烯; 自偶联反应; 钯催化剂

收稿日期: 2014-06-30. 接受日期: 2014-07-21. 出版日期: 2015-01-20.

*通讯联系人. 电话: (010)62759728; 传真: (010)62751708; 电子信箱: zfxi@pku.edu.cn

基金来源: 国家重点基础研究发展计划(973计划, 2012CB821600); 国家自然科学基金(21132001).

本文的英文电子版由Elsevier出版社在ScienceDirect上出版(http://www.sciencedirect.com/science/journal/18722067).

\section{1. 前言}

多取代环辛四烯及其衍生物(如二苯并环辛四烯)因 其独特的结构带来的特殊性质和反应性而成为一种重 要的有机合成中间体 ${ }^{[1-6]}$ 和金属配体 ${ }^{[7-10]}$. 目前已有一些 方法可以较高效地合成多取代环辛四烯 ${ }^{[11-17]}$, 但这些方
法大部分都需要使用当量的有机锂试剂 ${ }^{[15]}$ 或有机铜试 剂 ${ }^{[18-25]}$. 过渡金属催化的环辛四烯的合成多集中在炔

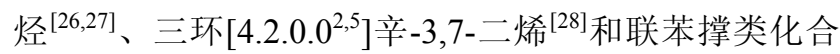
物 ${ }^{[29-35]}$ 的反应上.

烯基溴化物是一类重要的有机合成子 ${ }^{[34-56]}$. 其可通 过锂卤交换反应制备相应的有机锂试剂进而合成大 $\pi$ 共 
轭体系 ${ }^{[41-44]}$, 或通过过渡金属催化的胺化反应直接合成 吡咯衍生物 ${ }^{[45-50]}$. 本课题组和其他课题组发展了一系列 能高效合成1,4-二溴-1,3-丁二烯类化合物的方法 ${ }^{[35,50-52]}$, 并利用此类二溴化合物骨架的协同效应将其应用到新 型金属有机化合物和有机小分子化合物的合成 中 ${ }^{[22,53-56]}$.

从原理上看,利用官能团化(如金属化)的1,3-丁二烯 衍生物的自偶联反应来制备多取代环辛四烯是一个可 行的方法. 这种策略已经被Takahashi等 ${ }^{[18]}$ 、Itoh 等 ${ }^{[23,24]}$ 、 $\mathrm{Xi}$ 等 ${ }^{[25]}$ 和本课题组 ${ }^{[19-22,57]}$ 所采用(图式1). 本文发展了一 种利用钯催化二溴代化合物的[4+4]自偶联反应来合成 多取代环辛四烯和二苯并 $[a, e]$ 环辛四烯的方法(图式1).

\section{2. 实验部分}

\section{1. 试剂和仪器}

如无特别说明,所有原料均从试剂公司购得后未作 任何纯化处理直接使用,所有反应均采用标准Schlenk技 术在高纯氮气正压下或在Mikrouna Super (1220/750)手 套箱中进行. 手套箱中的氮气在铜/分子篮催化剂单元中 不断循环通过,并使用水氧联合分析仪进行监测, 以保证 水氧含量始终处于 $1 \mathrm{ppm}$ 以下. 溶剂四氢呋喃(THF)和甲 苯由Mbraun SPS-800溶剂处理系统除水除氧后加入钠丝 存放于手套箱保存. 柱层析所用石油醚沸程为 $60-90^{\circ} \mathrm{C}$. ${ }^{1} \mathrm{HNMR}$ 和 ${ }^{13} \mathrm{CNMR}$ 核磁共振谱由Bruker ARX400核磁共 振谱仪 $\left({ }^{1} \mathrm{H}\right.$ 谱 $400 \mathrm{MHz},{ }^{13} \mathrm{C}$ 谱 $\left.100 \mathrm{MHz}\right)$ 和Bruker ARX500 核磁共振谱仪 $\left({ }^{1} \mathrm{H}\right.$ 谱 $500 \mathrm{MHz},{ }^{13} \mathrm{C}$ 谱 $\left.125 \mathrm{MHz}\right)$ 在室温下测 得, 所用溶剂为氞代氯仿 $\left(\mathrm{CDCl}_{3}\right)$, 除特别说明外以四甲 基硅烷 $(0.00 \mathrm{ppm})$ 作为内标. 傅里叶变换高分辨质谱 (HMRS)测试分别采用Bruker Apex IV电喷雾电离(ESI) 和傅里叶变换离子回旋共振 (FT-ICR) 质谱仪, 以及 Bruker Daltonics Inc APEXII电子轰击(EI)和傅里叶变换 离子回旋共振(FT-ICR) 质谱仪. 气相色谱质谱联用仪 (GC/MS)检测采用电子轰击(EI)检测器.

\section{2. 二苯并环辛四烯和多取代环辛四烯的合成}

二溴化合物 $\mathbf{1}$ 和 2 采用文献报道的方法合成 ${ }^{[50-52]}$. 在 氮气保护下, 向 $25 \mathrm{~mL}$ 的Schlenk管中依次加入二溴化合 物( $0.6 \mathrm{mmol}, 2$ eqiuv.), $\mathrm{Pd}\left(t-\mathrm{Bu}_{3} \mathrm{P}\right)_{2}(0.015 \mathrm{mmol}, 5 \mathrm{~mol} \%)$, $t-\mathrm{Bu}_{3} \mathrm{P}(0.03 \mathrm{mmol}, 10 \mathrm{~mol} \%)$, $\operatorname{LiOEt}(1.2 \mathrm{mmol}, 4$ equiv. $)$ 和 $5 \mathrm{~mL}$ 甲苯. 将反应管置于 $120^{\circ} \mathrm{C}$ 油浴中搅拌反应 $24 \mathrm{~h}$ 后 将反应管冷却至室温. 反应混合物用水淬灭后使用乙酸 乙酯萃取, 得到的有机溶液在使用饱和 $\mathrm{NaCl}$ 溶液洗涤和 无水 $\mathrm{Na}_{2} \mathrm{SO}_{4}$ 干燥后减压蒸馏除去溶剂. 所得粗产物使用
硅胶柱色谱进行分离, 即可得到多取代环辛四烯产物 $\mathbf{3}$ 和 4 , 洗脱液为石油醚和乙酸乙酯混合溶剂.

\section{3. 二溴化合物 1 的表征数据}

(略,见英文部分)

\section{4. 多取代环辛四烯 3 和 4 的表征数据}

(略, 见英文部分).

\subsection{3c 的单晶结构表征}

将 $3 \mathbf{c}$ 的氛代氯仿溶液在室温下挥发 $1 \mathrm{~d}$, 可得到适合 $\mathrm{X}$ 射线单晶衍射结构测试的晶体. 该晶体用Rigaku CCD SATURN724型 X射线衍射仪在 $-98^{\circ} \mathrm{C}$ 采用经石墨单色 化的Mo $K_{a}$ 射线 $(\lambda=0.71073 \AA)$ 收集衍射数据. 结构用直 接法(SHELXTL-97)解出 ${ }^{[59,60]}$, 晶体结构修正用 olex2程 序 $^{[60,61]}$,氢原子坐标由差值Fourier合成法得到. 以上单晶 数据可通过剑桥晶体数据库 (www.ccdc.cam.ac.uk/ data_request/cif)进行检索. CCDC 1006078 (3c).

\section{3. 结果与讨论}

\section{1. 反应条件优化}

如表1所示, 本文首先选择 $1 \mathrm{a}$ 的反应作为模型反应 进行考察. 反应在特定条件下可以得到目标产物二苯并 $[a, e]$ 环辛四烯3a. 值得注意的是, 除了不同的催化剂和配 体在反应中所表现出来的差别外,碱的选择对该转化过 程十分敏感 ${ }^{[62]}$. 经笁选发现, LiOEt是最有效的碱, 使用 较强的碱(如 $\mathrm{NaO} t-\mathrm{Bu}$ 和 $\mathrm{KO} t-\mathrm{Bu}$ )会导致 $1 \mathbf{a}$ 分解, 而使用 较弱的碱(如 $\mathrm{NaOAc}$ ) 则不能得到相应产物 (表 1 , 实验 3-5). 反应中碱的用量也会显著影响 $\mathbf{3 a}$ 的产率 (表1, 实验 11 和15-18). 表 1 中还列出了其他一些反应条件的篮选 结果. 最终确定的最佳反应条件为: $\operatorname{Pd}\left(t-\mathrm{Bu}_{3} \mathrm{P}\right)_{2}(5 \mathrm{~mol} \%)$, $t-\mathrm{Bu}_{3} \mathrm{P}(10 \mathrm{~mol} \%), \operatorname{LiOEt}$ (4 equiv.) 在甲苯中 $120^{\circ} \mathrm{C}$ 反应 24 h. 此时相应产物 $\mathbf{3 a}$ 的分离收率可达 $83 \%$.

\section{2. 底物拓展}

在最优反应条件下,本文以中等至较好的收率合成 了一系列环辛四烯衍生物3a-3h (图式2). 底物中烯基上 的取代基对反应产率有很大影响. 当底物双键上为两个 烷基时, 可以高产率获得相应产物 3a-3c. 将底物双键上 的一个烷基换为苯基时,相应产物的产率会有明显降低 (3d). 底物中苯环上有不同取代基时也能顺利反应得到 相应的产物(3e-3g). 含有供电子基团的底物的反应产率 比含有吸电子基团的底物高. 四邻亚苯 $3 \mathbf{h}$ 也可以通过此 反应制备. 值得注意的是, 此反应有很高的选择性. 产物 $\mathbf{3 c}$ 的结构已经通过X射线单晶衍射确认(图1).

与此同时, 1,2,3,4-四乙基-1,4-二溴-1,3-丁二烯(2)也 
可以在此条件下顺利反应, 得到相应的环辛四烯产物 $3 \mathbf{i}$ (图式3).

\section{3. 机理研究}

为了阐明该自偶联反应的机理, 本文进行了一系列 的验证实验. (1) 在仅加入 0.5 当量LiOEt进行反应时, 只 能观察到痕量的 $3 a$ 生成, 同时伴随原料剩余; 增加 $\mathrm{LiOEt}$ 用量可有效提高 3a产率(表1, 实验11和15-18). (2) 文献 曾报道 ${ }^{[63,64]}$ 异丙醇可用作还原剂原位将反应体系中的 $\operatorname{Pd}(\mathrm{II})$ 物种还原为活性的 $\operatorname{Pd}(0)$ 物种, 同时自身被氧化为 丙酮. 本文作者使用气相色谱质谱联用仪对本自偶联反 应的气相和液相部分分别进行了原位检测, 发现在此反 应中有乙醛和乙酸乙酯生成 ${ }^{[65]}$. (3) 将反应中使用的碱 更换为 $\mathrm{LiO} t-\mathrm{Bu}$ 后, 则必须加入还原剂(如异丙醇) 该反应 才能顺利进行(表1, 实验13和14).

基于以上实验, 本文提出了以下可能的反应机 理 ${ }^{[57,66-70]}$ (图式4). 首先, $\operatorname{Pd}(0)$ 物种对底物1进行氧化加 成, 得到中间体 $\mathbf{5}$. 中间体 $\mathbf{5}$ 上的配体与 LiOEt中的乙氧基 进行配体交换反应后, 发生分子内迁移插入反应, 得到钯 杂环中间体6. 此钯杂环与底物再次发生氧化加成反应, 得到中间体 7. 中间体 7发生还原消除生成中间体 8 , 接着 发生第二次配体交换反应和分子内迁移插入反应, 得到
钯杂九元环中间体9. 最后, 中间体9发生还原消除反应 得到最终的环辛四烯产物 3 , 同时再生出活性 $\operatorname{Pd}(0)$ 物种.

本课题组曾经报道过一例钯催化含有烯基嗍官能 团的碘苯衍生物的自偶联环化生成二苯并 $[a, c]$ 环辛四 烯的反应 ${ }^{[57]}$ (图式 5 , 反应式 1 ). 为了研究这两个自偶联反 应的不同选择性, 作者进行了如下的对比实验. 当向反 应管中依次加入底物 $1 \mathbf{a}, \mathrm{PdCl}_{2}\left(\mathrm{PPh}_{3}\right)_{2}, i-\mathrm{PrOH}, \mathrm{Cs}_{2} \mathrm{CO}_{3}$ 和 甲苯进行反应, 能够同时观察到痕量的 $\mathbf{3 a}$ 和 $\mathbf{3 a}$ 两种产物 的生成, 同时原料 $1 \mathbf{a}$ 剩余(图式 5 , 反应式 2 ). 向此反应体 系中添加 $10 \mathrm{mmol} \%$ 的 $t-\mathrm{Bu}_{3} \mathrm{P}$ 进行反应, 则能观察到痕量 的 $3 \mathbf{a}^{2}$ 和 $10 \%$ 的 $\mathbf{3 a}$ 生成, 同时其余原料 $1 \mathbf{a}$ 剩余(表 1 , 实验 6). 这表明, 这两个反应中不同的选择性可能是配体的立 体效应造成的. 当反应中的碱选用 $\mathrm{Cs}_{2} \mathrm{CO}_{3}$ 时, $\mathrm{CO}_{3}{ }^{2-}$ 可以 作为配体与 $\mathrm{Pd}(\mathrm{IV})$ 金属中心配位, 使得相应的中间体 7, 与中间体 7的构型不相同, 进而导致了其后的还原消除 中的不同选择性(图式5).

\section{4. 结论}

本文发展了一种利用钯催化二溴代化合物的 [4+4] 自偶联反应合成环辛四烯衍生物的方法. 在此反应中, 配体的空间效应可能是影响反应区域选择性的原因. 